\title{
TRANSACTIONS OF THE
}

INTERNATIONAL ASTRONOMICAL

UNION

\section{VOL. XXIIIA (REPORTS 1996)}

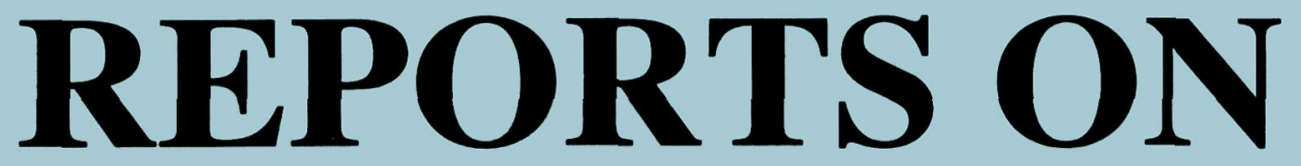

ASTRONOMY

KLUWER ACADEMIC PUBLISHERS

DORDRECHT / BOSTON / LONDON

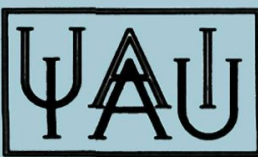




\section{REPORTS ON ASTRONOMY}

Transactions of the International Astronomical

Union, Volume XXIIIA

edited by

IMMO APPENZELLER

General Secretary of the Union

IAU Transactions are published as a volume corresponding to each General Assembly. Volume $\mathrm{A}$ is produced prior to the Assembly and contains Reports on Astronomy, prepared by each Commission President. The intention is to summarize the astronomical results that have affected the work of the Commission since the production of the previous Reports up to a time which is about one year prior to the General Assembly. Volume $B$ is produced after the Assembly and contains accounts of Commission Meetings which were held, together with other material. The reports included in the present volume range from outline summaries to lengthy compilations and references. Most of the reports are in English. 
INTERNATIONAL COUNCIL OF SCIENTIFIC UNIONS

INTERNATIONAL ASTRONOMICAL UNION

UNION ASTRONOMIQUE INTERNATIONALE

TRANSACTIONS

OF THE

INTERNATIONAL ASTRONOMICAL UNION

VOLUME XXIIIA

\section{REPORTS}

\section{ON}

\section{ASTRONOMY}

Edited by

IMMO APPENZELLER

General Secretary of the Union

KLUWER ACADEMIC PUBLISHERS

DORDRECHT / BOSTON / LONDON

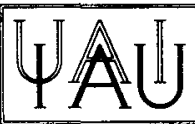


A.C.I.P. Catalogue record for this book is available from the Library of Congress

\author{
Published on behalf of \\ the International Astronomical Union \\ by \\ Kluwer Academic Publishers, P.O. Box 17, 3300 AA Dordrecht, The Netherlands. \\ Kluwer Academic Publishers incorporates \\ the publishing programmes of \\ D. Reidel, Martinus Nijhoff, Dr W. Junk and MTP Press. \\ Sold and distributed in the U.S.A. and Canada \\ by Kluwer Academic Publishers, \\ 101 Philip Drive, Norwell, MA 02061, U.S.A. \\ In all other countries, sold and distributed \\ by Kluwer Academic Publishers Group, \\ P.O. Box 322, 3300 AH Dordrecht, The Netherlands.
}

\title{
Printed on acid-free paper
}

\section{All Rights Reserved \\ (C)1997 International Astronomical Union}

No part of the material protected by this copyright notice may be reproduced or utilized in any form or by any means, electronic or mechanical including photocopying, recording or by any information storage and retrieval system, without written permission from the publisher.

Printed in the Netherlands 


\section{TABLE OF CONTENTS}

PREFACE

\section{INDIVIDUAL REPORTS OF DIVIONS/COMMISSIONS \& WORKING GROUPS}

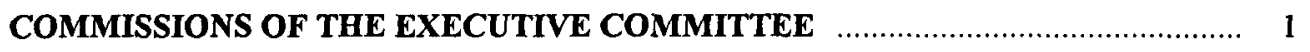

5 Documentation \& astronomical Data …........................................................ 3

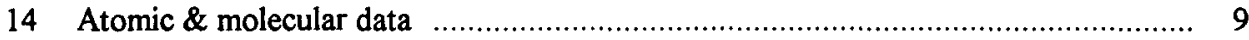

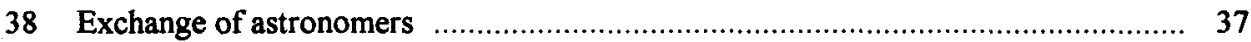

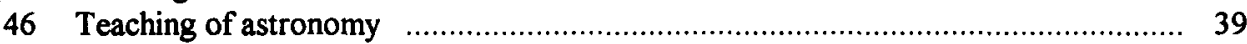

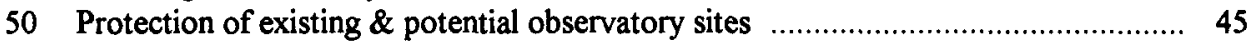

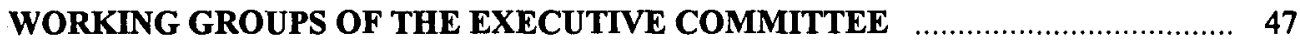

WG Encouraging the International Development of Antarctic Astronomy ................... 49

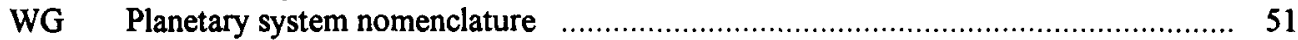

WG World wide Development of Astronomy f................................................. 57

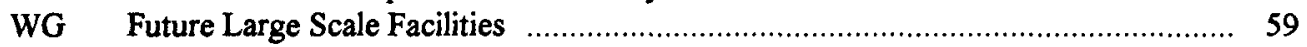

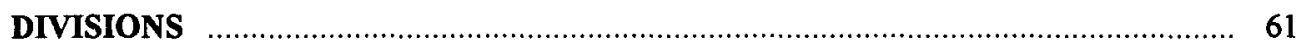

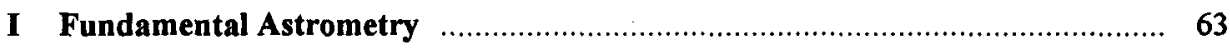

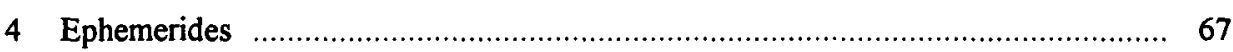

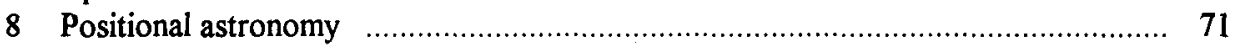

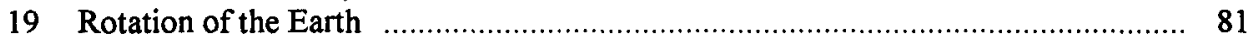

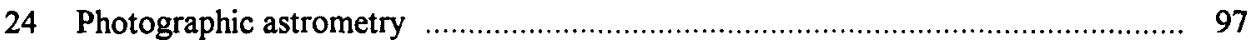

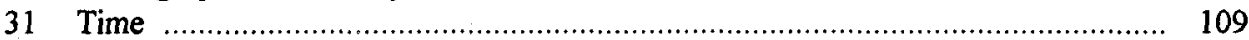

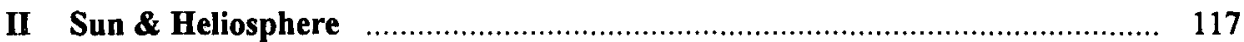

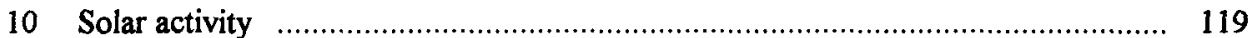

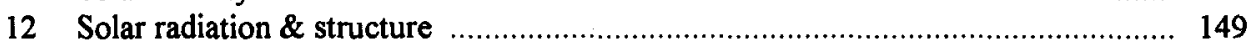

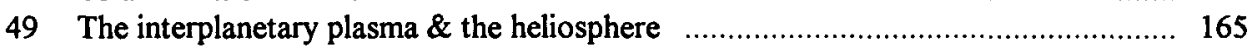

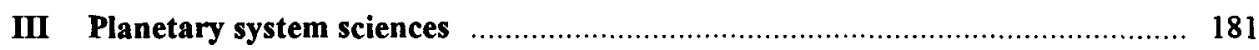

15 Physical study of comets, minor planets \& meteorites …................................ 183

16 Physical study of planets \& satellites ......................................................... 203

20 Position \& motions of minor planets, comets \& satellites f.............................. 215

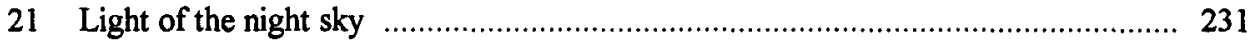

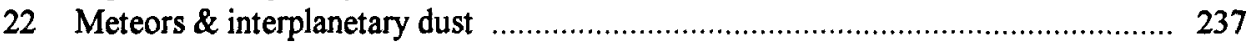

51 Bioastronomy: search for extraterrestrial life ........................................... 251

Inter-Commission WG on the prevention of interlanetary pollution $\ldots \ldots \ldots \ldots \ldots \ldots . . .263$ 


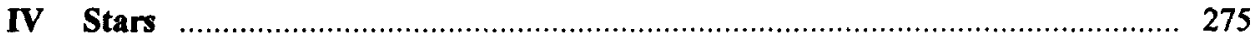

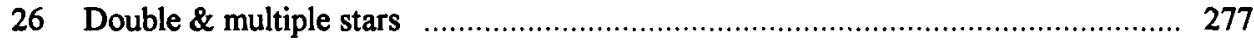

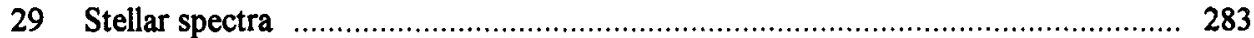

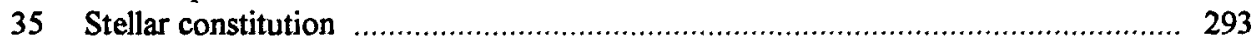

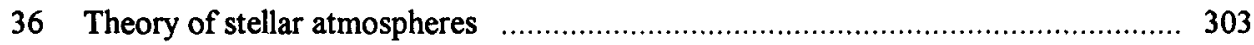

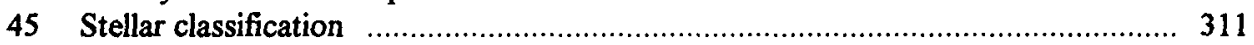

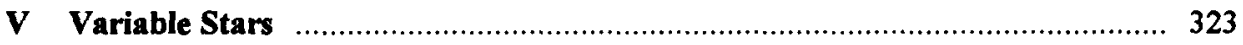

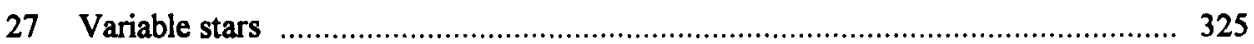

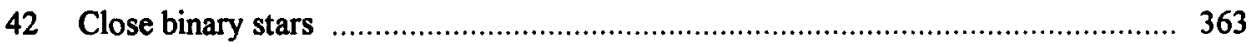

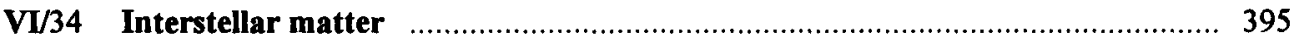

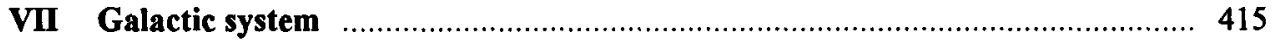

33 Structure \& dynamics of the galactic system ……….................................... 417

37 Star clusters \& associations Report not received

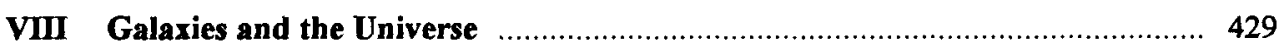

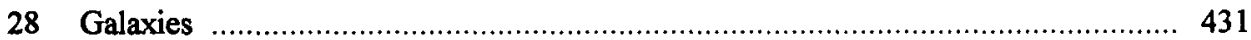

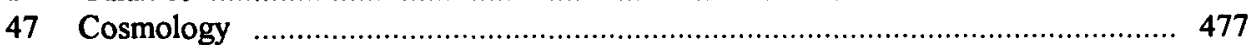

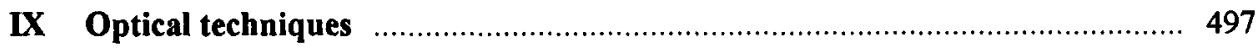

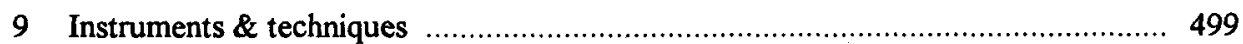

25 Stellar photometry \& polarimetry …………………................................... 515

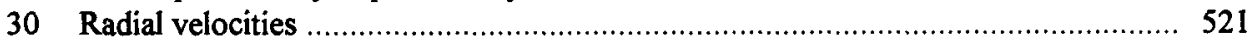

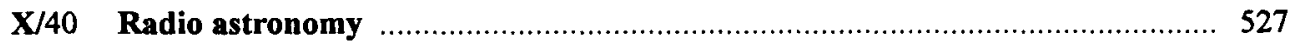

XI/44 Space and high energy astrophysics …………………………………..... 561

COMMISSIONS NOT ATTACHED TO A DIVISION ………………….... 585

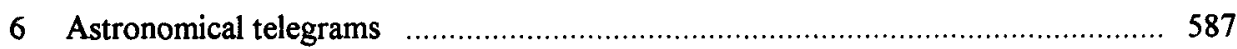

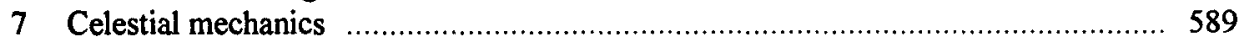

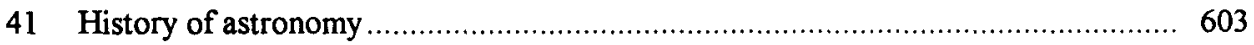

A Joint Commission of IAU \& IUHPS (International Union for History and Philosophy of Sciences) 